\title{
SEGURANÇA DO PACIENTE NA ATENÇÃO PRIMÁRIA À SAÚDE: REVISÃO INTEGRATIVA
}

\author{
Karina Oliveira de Mesquita ${ }^{1}$, Lielma Carla Chagas da Silva ${ }^{1}$, Roberta Cavalcante Muniz Lira ${ }^{2}$, CibellyAliny \\ Siqueira Lima Freitas ${ }^{3}$, Geison Vasconcelos Lira ${ }^{4}$
}

\begin{abstract}
RESUMO: Revisão integrativa que objetivou analisar produções científicas sobre a Segurança do paciente na Atenção Primária à Saúde. Realizada de janeiro a março de 2016, em periódicos nacionais, internacionais e nas bases de dados em ciências da saúde da Biblioteca Virtual em Saúde. Selecionaram-se dez artigos que respondiam à questão norteadora: de que forma é evidenciada na literatura a temática Segurança do Paciente na Atenção Primária à Saúde? Observou-se que as publicações datavam de 2008 em diante, com temática variando entre investigação da cultura de segurança, relação da segurança do paciente com a prevenção de eventos adversos e avaliação de incidentes, e tradução de instrumento para avaliação da segurança do paciente. O conteúdo evidenciado pela análise permitiu concluir sobre a importância de discutir sobre segurança de modo a contribuir na melhoria da qualidade da assistência à saúde, assim como a necessidade de novos estudos, haja vista relevância do tema e pouca produção científica na área.
\end{abstract}

DESCRITORES: Segurança do paciente; Atenção primária à saúde; Cuidados de enfermagem.

\section{PATIENT SAFETY IN PRIMARY HEALTH CARE: AN INTEGRATIVE REVIEW}

\begin{abstract}
This is an integrative review with the aim of analyzing scientific articlesaddressing patient safety in primary health care. It was conducted from January until March 2016 with national and international journals and with health sciences databases from the Virtual Library on Health. The author chose 10 articles that answered the guiding question: How does the literature depict the theme of patient safety in primary health care? With 2008 as the earliest publishing date, publication themes ranged from investigations on safety culture and the relation between patient safety and prevention of adverse events and incident assessment to translations of instruments for assessment of patient safety. It was possible to draw conclusions from content gathered during analysis about the importance of discussing safety to support the improvement of health care. The authors also found a need for more studies, considering the relevance of the theme and the limited scientific work on the theme.
\end{abstract}

DESCRIPTORS: Patient safety; Primary health care; Nursing care.

\section{SEGURIDAD DEL PACIENTE EN LA ATENCIÓN PRIMARIA DE SALUD: REVISIÓN INTEGRATIVA}

RESUMEN: Revisión integrativa objetivando analizar producciones científicas sobre Seguridad del Paciente en la Atención Primaria de Salud. Realizada de enero a marzo de 2016, en periódicos nacionales, internacionales y bases de datos de ciencias de salud de Biblioteca Virtual en Salud. Seleccionados diez artículos respondiendo la pregunta orientadora: "¿De qué forma está evidenciada en la literatura la temática Seguridad del Paciente en Atención Primaria de Salud?" Las publicaciones databan de 2008 en adelante, con temática variando entre investigación de cultura de seguridad, relación de seguridad del paciente con prevención de eventos adversos y evaluación de incidentes; y traducción de instrumento para evaluación de seguridad del paciente. El contenido analizado permitió determinar la importancia de discutir sobre seguridad apuntando a contribuir en mejorar la calidad de atención de salud, así como la necesidad de nuevos estudios, considerándose la relevancia del tema y la escasa producción científica en el área.

DESCRIPTORES: Seguridad del Paciente; Atención Primaria de Salud; Atención de Enfermería.

${ }^{1}$ Enfermeira. Mestranda em Saúde da Família. Universidade Federal do Ceará. Sobral, CE, Brasil.

${ }^{2}$ Enfermeira. Doutora em Saúde Pública. Docente da Universidade Federal do Ceará. Sobral, CE, Brasil.

${ }^{3}$ Enfermeira. Doutora em Enfermagem. Docente da Universidade Estadual Vale do Acaraú. Sobral, CE, Brasil.

${ }^{4}$ Médico. Doutor em Educação. Docente da Universidade Federal do Ceará. Sobral, CE, Brasil.

Autor Correspondente:

Karina Oliveira de Mesquita

Universidade Federal do Ceará

Av. Frederico Ferreira Gomes, 1175 - 62030-262 Sobral, CE, Brasil

E-mail: karinamesquita1991@gmail.com
Recebido: 01/03/2016

Finalizado: $12 / 05 / 2016$ 


\section{INTRODUÇÃO}

Segurança do paciente é um componente fundamental da qualidade de cuidados de saúde. Como as organizações de cuidado de saúde se esforçam continuamente para melhorar, há um reconhecimento crescente da importância de uma cultura de segurança do paciente ${ }^{(1)}$.

Nesta perspectiva, no inicio da última década do século XX a comunidade da saúde tem trazido para a pauta essa preocupação com a qualidade do cuidado e discussão sobre segurança do paciente. Muito disso a partir da divulgação do relatório intitulado To err is human: building a safer health care system, traduzindo "Errar é humano: a construção de um sistema de saúde mais seguro", publicado pelo Institute of Medicine (IOM), considerado marco para a segurança do paciente e datado deste mesmo período ${ }^{(2)}$.

Diante da mobilização mundial após a publicação do relatório, a Organização Mundial da Saúde (OMS) lançou a Aliança Mundial para a Segurança do Paciente em 2004. Isso despertou os países membros para o compromisso de desenvolver políticas públicas e práticas voltadas para a segurança do paciente, incluindo o Brasil $^{(3)}$.

A segurança consiste na redução do risco e danos desnecessários associados à assistência em saúde até um mínimo aceitável que, por sua vez, se refere àquilo que é viável diante do conhecimento atual, dos recursos disponíveis e do contexto em que a assistência foi realizada ${ }^{(4)}$.

Autores destacam que a maioria dos cuidados de saúde é desenvolvida fora dos ambientes hospitalares e que muitos incidentes identificados nos hospitais têm origem em outros locais, como na Atenção Primária à Saúde (APS) ${ }^{(5)}$.

Observa-se que as pesquisas relacionadas à segurança do paciente estão direcionadas principalmente para a assistência hospitalar, apesar da maioria dos pacientes receber seus cuidados de saúde na atenção primária, especialmente em países com um sistema de atenção primária forte e atuante ${ }^{(6-8)}$.

Apesar do crescente interesse na segurança dos pacientes, ainda é generalizada a falta de sensibilização para o problema. Nesse contexto, torna-se relevante o desenvolvimento de pesquisas relacionadas à temática, como forma de enriquecer e divulgar a literatura na área, reduzir as lacunas de conhecimento existentes e sensibilizar os profissionais sobre a importância da prática de segurança do paciente em todos os níveis de atenção à saúde, levando à redução dos riscos e danos relacionados à assistência.

Destaca-se ainda a Portaria $\mathrm{n}^{\circ} 529$ de $1^{\circ}$ de abril de 2013, do Ministério da Saúde, que institui o Programa Nacional de Segurança do Paciente, trazendo em seus objetivos específicos a necessidade de produzir, sistematizar e difundir conhecimentos sobre segurança do paciente e ampliar o acesso da sociedade às informações relativas a esse tema, justificando também a realização desta pesquisa ${ }^{(9)}$.

Dessa forma, o presente estudo tem o objetivo de analisar as produções científicas sobre a Segurança do paciente na Atenção Primária à Saúde.

\section{METODOLOGIA}

Realizou-se uma revisão integrativa da literatura. Essa possibilita a síntese do estado da arte do conhecimento de um determinado assunto de modo sistemático, apontando lacunas do conhecimento que precisam ser preenchidas com a realização de novos estudos como suporte à tomada de decisão e à melhoria da prática clínica, além de permitir a realização de uma síntese de múltiplos estudos publicados, viabilizando conclusões gerais a respeito de uma particular área de estudo ${ }^{(10)}$.

Para a elaboração da presente revisão integrativa, as seguintes etapas foram percorridas: estabelecimento da pergunta norteadora da revisão; estabelecimento de critérios de inclusão e exclusão de artigos; definição das informações a serem extraídas dos artigos selecionados; análise, discussão e apresentação dos resultados.

Formulou-se a seguinte questão norteadora para guiar a revisão: De que forma é evidenciada na literatura a temática de Segurança do Paciente na Atenção Primária à Saúde? 
Depois de formulada a questão estabeleceu-se três estratégias de busca, a primeira realizada nos periódicos nacionais, uma segunda em periódicos internacionais e a última na base de dados nacionais da Biblioteca Virtual em Saúde (BVS) por meio do cruzamento de descritores.

Para a seleção das produções, foram utilizados os seguintes critérios de inclusão para primeira e terceira estratégias de busca: artigos disponíveis em texto completo; artigos no idioma português, e que abordassem a temática em estudo. Para a segunda estratégia utilizaram-se os critérios estabelecidos para a primeira e terceira busca, excetuando o idioma que passaria para o inglês. Ao final das três buscas foram excluídas todas as produções duplicadas, editoriais e boletins epidemiológicos.

A primeira busca, realizada em janeiro de 2016, foi feita por meio do levantamento das produções relacionadas à temática "Segurança do Paciente na Atenção Primária à Saúde" em quinze periódicos nacionais: Acta Paulista de Enfermagem, Revista de Saúde Pública, Cadernos de Saúde Coletiva, Cadernos de Saúde Pública, Ciência \& Saúde Coletiva, Epidemiologia e Serviços de Saúde, Revista da Escola Anna Nery, Revista Interface, Revista Brasileira de Enfermagem, Revista da Escola de Enfermagem da USP, Revista Gaúcha de Enfermagem, Saúde e Sociedade, Saúde em debate, Texto Contexto Enfermagem e Physis. Nesta pesquisa foram identificados quatro artigos relacionados à temática, sendo dois publicados no periódico Cadernos de Saúde Pública, um na Revista Gaúcha de Enfermagem e um na Revista Texto Contexto Enfermagem.

A segunda busca feita no mês de fevereiro de 2016 foi realizada em nove periódicos internacionais, por meio da identificação de produções relacionadas à "Segurança do Paciente na Atenção Primária à Saúde", sendo eles: Canadian Medical Association Journal, The new England Journal of Medicine, International Journal for Quality in Health Care, BMJ Quality and Safety Health Care, The New Zealand Medical Journal, Administrative Science Quaterly, Management Science, Journal of Nursing Scholarship e Medical Journal of Australia. Foram identificados cinco artigos relacionados ao tema.

Por último, em março de 2016, foi feita uma pesquisa bibliográfica na BVS, com produções indexadas nas bases de dados: Scielo, Lilacs, Medline e BDENF. Para isso, foram cruzados os descritores "Segurança do paciente" e "Atenção Primária à Saúde", consultados nos Descritores em Ciências da Saúde (DECS). O cruzamento foi feito com o operador booleano and. A busca feita na BVS resultou em 139 produções. Após seleção minuciosa pelos critérios de inclusão e exclusão, resultou-se em quatro artigos para análise.

As produções resultantes das buscas bibliográficas totalizaram em dez, e estas foram lidas e analisadas descritivamente sendo dispostas em quadros considerando aspectos relevantes.

\section{- RESULTADOS}

Considerando a proporção de artigos publicados e utilizados na revisão, identifica-se que a produção científica relacionada à temática de segurança do paciente tem sido crescente nos últimos anos, acompanhando as discussões e intencionalidades da OMS. De modo que inicialmente tenha sido evidenciada nas publicações internacionais, nos anos de 2008 e 2009, aparecendo na literatura nacional a partir de 2011, como pode ser observado no Quadro 1 a seguir.

Dos dez artigos analisados, predominaram os publicados no periódico internacional "International Journal for Quality in Health Care" com o total de quarto artigos, seguido do "Cadernos de Saúde Pública" com dois artigos, e os demais: Revista da Escola de Enfermagem da USP, Texto Contexto Enfermagem, Revista Gaúcha de Enfermagem, The New Zealand Medical Journal com uma publicação cada.

No que refere à tipologia do estudo, predominaram as pesquisas do tipo transversal (quatro), sejam eles epidemiológicos ou prospectivos, seguido dos estudos descritivos (dois), estudos de revisão (dois), estudo teórico (um) e estudo seccional (um), conforme apresentado no Quadro 2.

Com relação à temática dos artigos, três faziam menção à investigação da cultura de segurança, os demais (seis) versavam entre a relação da temática segurança do paciente com a prevenção de eventos adversos e avaliação de incidentes, excetuando um estudo que tratava da tradução e adaptação de um questionário. 
Quadro 1 - Apresentação dos artigos científicos selecionados por ano de publicação. Sobral, CE, Brasil, 2016

\begin{tabular}{|l|l|}
\hline Título & Ano \\
\hline Using nurses and office staff to report prescribing errors in primary care & 2008 \\
\hline A survey on patient safety culture in primary healthcare services in Turkey & 2009 \\
\hline Qualidade da higienização das mãos de profissionais atuantes em Unidades Básicas de Saúde & 2011 \\
\hline Cultura da segurança do paciente na Atenção Primária à Saúde & 2013 \\
\hline Safety climate and its association with office type and team involvement in primary care & 2013 \\
\hline O papel da atenção primária na prevenção de infecções relacionadas à assistência à saúde & 2014 \\
\hline Segurança do paciente na atenção primária à saúde: revisão sistemática & 2014 \\
\hline Exploring patient safety culture in primary care & 2014 \\
\hline $\begin{array}{l}\text { Using triggers in primary care patient records to flag increased adverse event risk and measure } \\
\text { patient safety at clinic level }\end{array}$ & 2014 \\
\hline $\begin{array}{l}\text { Tradução e adaptação de um questionário elaborado para avaliar a segurança do paciente na } \\
\text { atenção primária em saúde }\end{array}$ & 2015 \\
\hline
\end{tabular}

\section{- DISCUSSÃO}

A análise da produção científica revelou que inúmeras organizações têm se dedicado a avaliar a ocorrência de incidentes relacionados ao cuidado em saúde, visando melhorar a qualidade da assistência prestada. De modo que, há dois anos, a OMS constituiu um grupo para estudar os riscos e os incidentes na Atenção Primária em Saúde ${ }^{(20)}$.

Considerando o período das publicações nesta revisão, que traduzem as iniciativas tomadas pioneiramente por países estrangeiros, destacam-se os Estados Unidos da América e vários outros países com configurações de sistemas de saúde distintos, dentre eles Inglaterra, Irlanda, Austrália, Canadá, Espanha, França, Nova Zelândia e Suécia, que protagonizaram iniciativas como a criação de institutos, associações e organizações voltadas para o campo da segurança do paciente(21).

Em estudo que buscava analisar a segurança do paciente no âmbito na APS com enfoque na prevenção de Infecções Relacionadas à Assistência a Saúde (IRAS), foi possível evidenciar que a APS possui um papel indireto na prevenção de IRAS, dado que deve atuar na prevenção de enfermidades e, consequentemente, na redução de internações hospitalares desnecessárias. Além disso, trás em discussão o fenômeno caracterizado como hospitalocentrismo, que enfatiza de maneira desproporcional a resolução dos problemas de saúde por meio do atendimento hospitalar. Considerando que internações hospitalares causam muitos mais custos à instituição, levantam-se as questões sobre qual seria a real necessidade dessas internações? Elas poderiam ser evitadas nos outros níveis de atenção? ?(16) $^{(1)}$

Os mesmos autores destacam que, além da prevenção das Internações por Condições Sensíveis à Atenção Primária (ICSAP) e do acesso oportuno, a APS deve atuar também no sistema de referência e contra-referência e pode contribuir com a vigilância pós-alta de IRAS. Em suma, embora o princípio de não causar dano esteja intrinsecamente ligado ao papel da APS, pela inerente potencialidade deste nível de atenção para a qualificação da saúde, é necessário ampliar a reflexão de que a segurança em saúde não começa apenas quando um indivíduo é hospitalizado ${ }^{(16)}$.

Os riscos associados aos procedimentos mais comuns realizados na APS são pouco conhecidos, com exceção daqueles referentes à imunização. Comumente são realizados na APS procedimentos com certo grau de invasividade, como coleta de exames colpocitológico, inserção de dispositivo intrauterino (DIU), cauterização de colo de útero, glicemia capilar, inalações, curativos, administração de injetáveis, para os quais se podem antecipar algum evento adverso associado. Entretanto, até o momento não existem estimativas a este respeito. Quanto aos procedimentos odontológicos, é bem reconhecido o risco associado à aquisição de vírus (ex.: hepatite $\mathrm{B}, \mathrm{HIV}$ ), porém são raros os relatos de IRAS causadas por bactérias ${ }^{(16)}$. 
Quadro 2 - Distribuição dos dados de identificação dos artigos científicos. Sobral, CE, Brasil, 2016

\begin{tabular}{|c|c|c|c|c|}
\hline Artigo & Periódico & Autores & Objetivo & Tipo de Estudo \\
\hline $\begin{array}{l}\text { Using nurses and office } \\
\text { staff to report } \\
\text { prescribing errors in } \\
\text { primary care }\end{array}$ & $\begin{array}{l}\text { International } \\
\text { Journal for } \\
\text { Quality in } \\
\text { Health Care }\end{array}$ & $\begin{array}{l}\text { Kennedy AG, } \\
\text { Littenberg B, } \\
\text { Senders JW }{ }^{(11)}\end{array}$ & $\begin{array}{c}\text { Implementar um sistema de } \\
\text { comunicação de prescrição de } \\
\text { erros em cuidados primários e } \\
\text { analisar os relatórios } \\
\end{array}$ & $\begin{array}{c}\text { Estudo } \\
\text { descritivo }\end{array}$ \\
\hline $\begin{array}{c}\text { A survey on patient } \\
\text { safety culture in primary } \\
\text { healthcare services in } \\
\text { Turkey }\end{array}$ & $\begin{array}{l}\text { International } \\
\text { Journal for } \\
\text { Quality in } \\
\text { Health Care }\end{array}$ & $\begin{array}{c}\text { Bodur S, Filiz } \\
E^{(12)}\end{array}$ & $\begin{array}{l}\text { Avaliar a cultura de segurança } \\
\text { do paciente em unidades } \\
\text { cuidados de saúde primários }\end{array}$ & $\begin{array}{l}\text { Estudo } \\
\text { seccional }\end{array}$ \\
\hline $\begin{array}{c}\text { Qualidade da higienização } \\
\text { das mãos de } \\
\text { profissionais atuantes } \\
\text { em Unidades Básicas de } \\
\text { Saúde }\end{array}$ & $\begin{array}{c}\text { Revista } \\
\text { Gaúcha de } \\
\text { Enfermagem }\end{array}$ & $\begin{array}{c}\text { Locks L, } \\
\text { Lacerda JT, } \\
\text { Gomes E, } \\
\text { Serratine } \\
\text { ACP }^{(13)}\end{array}$ & $\begin{array}{c}\text { Avaliar a qualidade de } \\
\text { higienização das mãos de } \\
\text { profissionais }\end{array}$ & $\begin{array}{l}\text { Estudo } \\
\text { epidemioló- } \\
\text { gico } \\
\text { transversal }\end{array}$ \\
\hline $\begin{array}{l}\text { Cultura da segurança } \\
\text { do paciente na Atenção } \\
\text { Primária à Saúde }\end{array}$ & $\begin{array}{c}\text { Texto } \\
\text { Contexto } \\
\text { Enfermagem }\end{array}$ & $\begin{array}{l}\text { Paese F, } \\
\text { Dal Sasso } \\
\text { GTM }^{(14)}\end{array}$ & $\begin{array}{l}\text { Analisar as atitudes que } \\
\text { evidenciam a cultura da } \\
\text { segurança do paciente pelos } \\
\text { profissionais das equipes da } \\
\text { Estratégia Saúde da Família } \\
\text { e do programa de agentes } \\
\text { comunitários de saúde }\end{array}$ & $\begin{array}{c}\text { Estudo } \\
\text { transversal } \\
\text { prospectivo }\end{array}$ \\
\hline $\begin{array}{l}\text { Safety climate and its } \\
\text { association with office } \\
\text { type and team } \\
\text { involvement in primary } \\
\text { care }\end{array}$ & $\begin{array}{l}\text { International } \\
\text { Journal for } \\
\text { Quality in } \\
\text { Health Care }\end{array}$ & \begin{tabular}{|c|} 
Gehring K, \\
Schwappach \\
DLB, Battaglia \\
M, Buff R, \\
Huber F, Sauter \\
P, et al ${ }^{(15)}$ \\
\end{tabular} & $\begin{array}{c}\text { Avaliar diferentes percepções } \\
\text { do clima de segurança entre } \\
\text { grupos de profissionais de } \\
\text { saúde e tipos de organizações } \\
\text { de cuidados primários em } \\
\text { saúde } \\
\end{array}$ & $\begin{array}{l}\text { Estudo } \\
\text { transversal }\end{array}$ \\
\hline $\begin{array}{c}\text { O papel da Atenção } \\
\text { Primária na prevenção de } \\
\text { infecções relacionadas à } \\
\text { assistência à saúde }\end{array}$ & $\begin{array}{l}\text { Revista da } \\
\text { Escola de } \\
\text { Enfermagem } \\
\text { da USP }\end{array}$ & \begin{tabular}{|l} 
Padoveze MC, \\
Figueiredo \\
RM $^{(16)}$
\end{tabular} & $\begin{array}{c}\text { Desenvolver um estudo } \\
\text { teórico na perspectiva do } \\
\text { principio primum non nocere, } \\
\text { analisando a segurança do } \\
\text { paciente na atenção à saúde } \\
\text { no âmbito da APS com } \\
\text { enfoque na prevenção de } \\
\text { IRAS }\end{array}$ & Estudo teórico \\
\hline $\begin{array}{l}\text { Segurança do paciente na } \\
\text { atenção primária à saúde: } \\
\text { revisão sistemática }\end{array}$ & \begin{tabular}{|l|} 
Cadernos de \\
Saúde Pública
\end{tabular} & \begin{tabular}{|c|} 
Marchon SG, \\
Mendes Junior \\
WV $^{(17)}$
\end{tabular} & $\begin{array}{c}\text { Identificar metodologias } \\
\text { utilizadas para avaliação de } \\
\text { incidentes na atenção primária } \\
\text { à saúde }\end{array}$ & $\begin{array}{c}\text { Revisão } \\
\text { sistemática }\end{array}$ \\
\hline $\begin{array}{l}\text { Exploring patient safety } \\
\text { culture in primary care }\end{array}$ & $\begin{array}{l}\text { International } \\
\text { Journal for } \\
\text { Quality in } \\
\text { Health Care }\end{array}$ & $\begin{array}{c}\text { Verbakel NJ, } \\
\text { Melle MV, } \\
\text { Langelaan M, } \\
\text { Verheij TJM, } \\
\text { Wagner C, } \\
\text { Zwart DLM } \\
\text { Zw8) }\end{array}$ & $\begin{array}{l}\text { Explorar as percepções sobre } \\
\text { a cultura de segurança em } \\
\text { nove tipos diferentes de } \\
\text { profissões da atenção primária } \\
\text { e suas possíveis diferenças }\end{array}$ & $\begin{array}{l}\text { Estudo } \\
\text { transversal }\end{array}$ \\
\hline $\begin{array}{l}\text { Using triggers in primary } \\
\text { care patient records to } \\
\text { flag } \\
\text { increased adverse event } \\
\text { risk and measure patient } \\
\text { safety at clinic level }\end{array}$ & $\begin{array}{l}\text { The New } \\
\text { Zealand } \\
\text { Medical } \\
\text { Journal }\end{array}$ & $\begin{array}{l}\text { Eggleton KS, } \\
\text { Dovey SM }{ }^{(19)}\end{array}$ & $\begin{array}{l}\text { Estabelecer qual ferramenta } \\
\text { de gatilho para eventos } \\
\text { adversos funcionou, qual a } \\
\text { mais útil e qual poderia ser } \\
\text { utilizada rotineiramente }\end{array}$ & $\begin{array}{l}\text { Revisão de } \\
\text { literatura }\end{array}$ \\
\hline $\begin{array}{l}\text { Tradução e adaptação } \\
\text { de um questionário } \\
\text { elaborado para avaliar a } \\
\text { segurança do paciente } \\
\text { na atenção primária em } \\
\text { saúde }\end{array}$ & \begin{tabular}{|c|} 
Cadernos de \\
Saúde Pública
\end{tabular} & $\begin{array}{c}\text { Marchon SG; } \\
\text { Mendes Junior } \\
\text { WV }^{(20)}\end{array}$ & $\begin{array}{c}\text { Descrever as etapas de } \\
\text { tradução e adaptação do } \\
\text { questionário do Primary Care } \\
\text { International Study of Medical } \\
\text { Errors (PCISME) }\end{array}$ & $\begin{array}{c}\text { Estudo } \\
\text { descritivo }\end{array}$ \\
\hline
\end{tabular}


Outra pesquisa investigou sobre a realização da higienização das mãos em Unidades Básicas de Saúde, sendo considerado como necessário, já que no Brasil a maioria dos estudos desse cunho é realizada apenas nos hospitais. Neste estudo pode-se perceber que os profissionais em sua grande maioria não realizavam a higienização das mãos corretamente e, quando o faziam, grande parte não seguia a técnica certa. Observa-se aí a negligência no procedimento sabidamente essencial no controle das infecções cruzadas. Ressalta-se ainda a escolha das IRAS, pela OMS, como primeiro desafio global para segurança do paciente tendo como ação chave a promoção da higienização das mãos ${ }^{(13)}$.

Um estudo de revisão sistemática afirma que, embora a maioria dos cuidados seja prestada em nível da atenção primária à saúde, as investigações sobre a segurança dos pacientes têm sido centradas em hospitais. Tendo em vista que os cuidados hospitalares são mais complexos, é natural que este ambiente seja o foco dessas pesquisas ${ }^{(17)}$.

Foram identificados como os fatores contribuintes de incidentes: falhas na comunicação interprofissional e com o paciente; falhas na gestão, tais como: falta de insumos medico-cirúrgicos e de medicamentos, profissionais pressionados para serem mais produtivos em menos tempo, falhas em prontuários, falhas na recepção dos pacientes, planta física da unidade de saúde inadequada, descarte inadequado de resíduos da unidade de saúde, tarefas excessivas e falhas no cuidado ${ }^{(17)}$.

Este mesmo estudo apresenta várias soluções para esses fatores, tais como: divulgar as práticas seguras; adequar as estruturas físicas; capacitar os profissionais da equipe de saúde; melhorar a comunicação interprofissional; melhorar a gestão das unidades de saúde, permitir que os pacientes e os profissionais reconheçam e gerenciem os eventos adversos; capacitar o profissional de saúde para compartilhar mudanças na equipe, para identificar e atuar nas situações de riscos; motivar os profissionais de saúde para agir em prol da segurança do paciente; participar (o profissional de saúde) nas decisões gerenciais; criar sistemas de avaliações de desempenho do médico ${ }^{(17)}$.

Reitera-se a necessidade de ampliar a cultura de segurança na atenção primária à saúde, a fim de habilitar pacientes e profissionais para reconhecer e gerenciar os eventos adversos, sendo sensíveis à sua capacidade compartilhada para a mudança, reduzindo erros e tensões entre profissionais e população ${ }^{(17)}$.

No que refere à cultura de segurança do paciente na APS, foi possível observar o erro como uma atitude menos relevante para este contexto. De certa forma, o erro ainda está muito associado à culpa, a um ambiente de trabalho punitivo e a uma cultura de pensar que os erros provocados pelo prestador de cuidado em saúde é resultado de descuido. Assim, trabalhar nas equipes sobre o erro e a culpa pode ser uma alternativa para modificar e transformar o erro em oportunidade de discutir e desenvolver o pensar crítico sobre as ações de cuidado e as atitudes frente ao próprio erro e ao erro do colega, ou seja, percebê-lo como oportunidade de aprendizado para impedir novos eventos relacionados à mesma causa ${ }^{(14)}$.

Estudo realizado na Turquia reforça o exposto anteriormente ao evidenciar que melhorar a cultura de segurança do paciente deve ser uma prioridade entre os administradores dos centros de saúde, e que profissionais de saúde devem ser encorajados a reportar erros sem medo de ação punitiva. Os autores corroboram citando dimensões consideradas essenciais para a avaliação da cultura de segurança do paciente, sendo elas: liderança, políticas e procedimentos, recursos humanos, comunicação e apresentação ${ }^{(12)}$.

Outro aspecto merecedor de destaque é que o clima de segurança do paciente na atenção primária sofre influência da qualidade do vínculo entre a equipe em reuniões envolvendo todos os membros, sendo esta uma maneira eficaz de fortalecer o clima de segurança em termos de estratégias baseadas em equipe e atividades de prevenção de $\operatorname{erros}^{(15)}$.

Em um estudo que investigou erros de prescrição de medicamentos em serviços de cuidados primários de uma cidade dos EUA sob a visão de enfermeiros e pessoal de escritório, foi destacada a importância dos relatos desses profissionais, tido como recursos valiosos em pesquisas desse cunho ${ }^{(11)}$. Estudo realizado na Holanda já destaca a importância da variedade de profissões que atuam na atenção primária para contribuir na implementação de estratégias de segurança ${ }^{(18)}$.

Outro estudo traz à tona outra importante discussão, que é a tradução de instrumentos como o 
questionário Primary Care International Study of Medical Errors (PCISME) que possam auxiliar nas pesquisas de segurança do paciente a nível nacional, refletindo da sua contribuição em mensurar incidentes em APS. Além disso, a aplicação desses instrumentos chama a atenção para o problema da ocorrência de danos em pacientes na APS ${ }^{(20)}$.

\section{- CONSIDERAÇÕES FINAIS}

Este estudo apresentou evidências científicas sobre o fenômeno da segurança do paciente na APS, trazendo desde a tradução para utilização de instrumentos que possam avaliar esta segurança até medidas de prevenção e aspectos culturais empregados na temática.

Permitiu ainda observar a escassez de produções científicas relacionadas à segurança do paciente na APS, nível de atenção este em que também podem ocorrer erros relacionados à assistência à saúde que causam algum dano ao paciente. Nesse contexto, muitas lacunas do conhecimento perpassam o estudo da temática, tais como as poucas informações acerca da cultura de segurança entre os profissionais da APS, a difícil mensuração dos tipos de incidentes ocorridos nesse nível de atenção, a escassez de estratégias para abordagem e sensibilização da temática e de instrumentos para investigações desse cunho.

Anseia-se que esta pesquisa suscite a elaboração de novos estudos sobre a temática, de maneira que possam ser construídas novas estratégias de sensibilização e divulgação da prática entre os profissionais em todos os âmbitos da assistência à saúde.

\section{- REFERÊNCIAS}

1. Sorra J, Famolaro T, Dyer N, Nelson d, Khanna K. Hospital survey on patient safety culture. Rockville (US): Agency for Healthcare Research and Quality; 2009.

2. Kohn LT, Corrignan JM, Donaldson MS. To err is human: building a safer health system. $2^{\mathrm{a}}$ ed. Washington: National Academy Press; 1999.

3. Capucho HC, Cassiani SHB. Necessidade de implantar programa nacional de segurança do paciente no Brasil. Rev. Saúde Pública. 2013; 47(4): 791-8.

4. da Silva LD, Camerini FG. Análise da administração de medicamentos intravenosos em hospital da rede sentinela. Texto Contexto Enferm. [Internet] 2012; 21(3) [acesso em 01 mar 2016]. Disponível: http://dx.doi. org/10.1590/S0104-07072012000300019.

5. Makeham M, Dovey S, Runciman W, Larizgoitia I. Methods and measures used in primary care patient safety research. Geneva: World Health Organization; 2008.

6. Donaldson SL. An international language for patient safety: Global progress in patient safety requires classification of key concepts. Int J Qual Health Care. [Internet] 2009; 21(1) [acesso em 23 nov 2015]. Disponível: http://dx.doi.org/10.1093/intqhc/mzn056.

7. Starfield B, Shi L, Macinko J. Contribution of primary care to health systems and health. Milbank Q. 2005; 83(3): 457-502.

8. Stelfox HT, Palmisani S, Scurlock C, Orav EJ, Bates DW. The "To Err is Human" report and the patient safety literature. Qual Saf Health Care. 2006; 15(3): 174-8.

9. Ministério da Saúde (BR). Portaria n. 529, de $1^{\circ}$ de abril de 2013. Institui o Programa Nacional de Segurança do Paciente (PNSP). [Internet] Brasília (DF): Ministério da Saúde; 2013 [acesso em 23 nov 2015]. Disponível: http:// bvsms.saude.gov.br/bvs/saudelegis/gm/2013/prt0529_01_04_2013.html.

10. Polit DF, Beck CT. Using research in evidence-based nursing practice. In: Polit DF, Beck CT, editors. Essentials of nursing research. Methods, appraisal and utilization. Philadelphia (USA): Lippincott Williams \& Wilkins; 2011. 
11. Kennedy AG, Littenberg B, Senders JW. Using nurses and office staff to report prescribing errors in primary care. Int J Qual Health Care. 2008; 20(4): 238-45.

12. Bodur S, Filiz E. A survey on patient safety culture in primary healthcare services in Turkey. Int J Qual Health Care. 2009; 21(5): 348-55.

13. Locks L, Lacerda JT, Gomes E, Serratine ACP. Qualidade da higienização das mãos de profissionais atuantes em Unidades Básicas de Saúde. Rev. Gaúcha Enferm. 2011; 32(3): 569-75.

14. Paese F, Dal Sasso GTM. Cultura da segurança do paciente na atenção primária à saúde. Texto Contexto Enferm. 2013; 22(2): 302-10.

15. Gehring K, Schwappach DLB, Battaglia M, Buff R, Huber F, Sauter P, et al. Safety climate and its association with office type and team involvement in primary care. Int J Qual Health Care. 2013; 25(4): 394-402.

16. Padoveze MC, de Figueiredo RM. O papel da Atenção Primária na prevenção de Infecções Relacionadas à Assistência à Saúde. Rev. esc. enferm. USP. 2014; 48(6): 1137-44.

17. Marchon SG, Mendes Junior WV. Segurança do paciente na atenção primária à saúde: revisão sistemática. Cad. Saúde Pública. 2014; 30(9): 1815-35.

18. Verbakel NJ, Melle MV, Langelaan M, Verheij TJM, Wagner C, Zwart DLM. Exploring patient safety culture in primary care. Int J Qual Health Care. 2014; 26(6): 585-91.

19. Eggleton KS, Dovey SM. Using triggers in primary care patient records to flag increased adverse event risk and measure patient safety at clinic level. N Z Med J. 2014; 127(1390): 45-52.

20. Marchon SG, Mendes Junior WV. Questionário para avaliar a segurança do paciente na Atenção Primária em Saúde. Cad. Saúde Pública. 2015; 31(7): 1395-402.

21. Reis CT, Martins M, Laguardia J. A segurança do paciente como dimensão da qualidade do cuidado de saúde: um olhar sobre a literatura. Ciênc. saúde coletiva. 2013; 18(7): 2029-36. 